\title{
Journal Watch: our experts look back and highlight some of the key breast cancer research articles from 2018
}

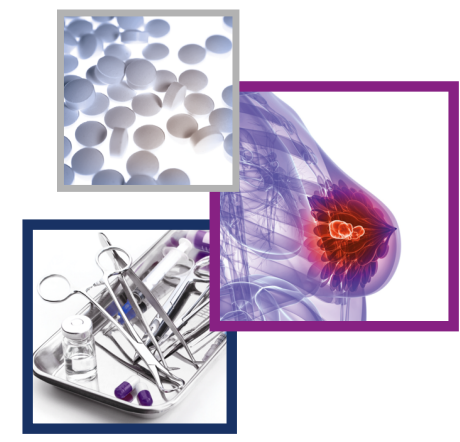

\author{
Elaine M Walsh ${ }^{1}$, Tadahiko Shien² \& Cesar A Santa-Maria*,1 \\ ${ }^{1}$ Breast and Ovarian Cancer Program, Sidney Kimmel Comprehensive Cancer Center, Johns Hopkins Hospital, MD, 21287, USA \\ ${ }^{2}$ Department of Breast \& Endocrine Surgery, Okayama University Hospital, Japan \\ *Author for correspondence: csantam2@jhmi.edu
}

First draft submitted: 14 December 2018; Accepted for publication: 17 December 2018; Published online: 1 March 2019

Loibl S, O'Shaughnessy J, Untch M, et al. Addition of the PARP inhibitor veliparib plus carboplatin or carboplatin alone to standard neoadjuvant chemotherapy in triple-negative breast cancer (BrighTNess): a randomised, Phase III trial. Lancet Oncol. 19(4), 497-509 (2018). There is a growing body of evidence for the use of PARP inhibitors in triple-negative breast cancer (TNBC). Many TNBCs harbor germline BRCA1/2 mutations, wild-type TNBCs display features of 'BRCAness' and there are biological similarities between TNBC and epithelial ovarian cancers. In the Phase III BrighTNess study, carboplatin \pm veliparib were added to anthracycline-taxane based neoadjuvant chemotherapy in Stage II-III TNBCs. The pCR rates were higher among patients treated with carboplatin compared with standard chemotherapy (58 vs $31 \%$, respectively; $\mathrm{p}<0.0001)$, but the addition of veliparib to carboplatin did not further increase pCR rates $(53$ vs $58 \%$, respectively; $\mathrm{p}=0.36$ ). Similarly, among $B R C A 1 / 2$ mutation carriers, there was no benefit from the addition of veliparib, although patient numbers were small. While this study demonstrated higher $\mathrm{pCR}$ rates with carboplatin-based chemotherapy in the treatment of TNBCs, it demonstrates that PARP inhibitors do not increase responses in unselected patients.

- Written by Elaine M Walsh and Cesar A Santa-Maria

\section{Litton JK, Rugo HS, Ettl J, et al. Talazoparib in patients with advanced breast cancer \& a} germline BRCA mutation. N. Engl. J. Med. 379(8), 753-763 (2018).

The EMBRACA study was a randomized Phase III study of talazoparib versus physician's choice (capecitabine, eribulin, gemcitabine or vinorelbine) in patients with metastatic BRCA1/2-mutated HER2-negative breast cancer $(\mathrm{BC})$, treated with $\leq 3$ lines of chemotherapy. Prior adjuvant/neoadjuvant platinum-based chemotherapy was allowed if a disease-free interval of at least 6 months had passed; progression on platinum for metastatic disease was excluded. The median progression-free survival favored talazoparib (8.6 months) versus chemotherapy (5.6 months) and response rates were higher (63 vs $27 \%$, respectively). The OlympiAD study, which investigated olaparib, has similar results. These studies highlight the importance of patient selection and demonstrate that the benefit of PARP inhibition in BC may be limited to those with BRCA1/2 mutations. To expand on genomic selection, the TBCRC048 study, a Phase II study of olaparib in patients with metastatic BC with either germline or somatic mutations in DNA repair genes, is currently open and recruiting patients (NCT03344965).

- Written by Elaine M Walsh and Cesar A Santa-Maria

\section{Banda K, Swisher EM, Wu D, Pritchard CC, Gadi VK. Somatic reversion of germline BRCA2} mutation confers resistance to poly(ADP-ribose) polymerase inhibitor therapy. JCO Precis. Oncol. (2), 1-6 (2018).

Despite the efficacy of PARP inhibition in patients with BRCA1/2 mutations, patients eventually develop resistance to therapy. The precise mechanisms of resistance to PARP inhibition remain unknown; acquired secondary mutations that restore the BRCA open reading frame and reversion mutations that restore the wild-type BRCA gene are two plausible explanations. This case report describes a patient with metastatic BRCA2-mutated $\mathrm{BC}$ who received a PARP inhibitor for 5 months before developing PARP resistance with progressive disease. Whole exome deep tissue

Future Medicine 
sequencing of progressive disease demonstrated the restoration of wild-type $B R C A 2$ function. This suggests that a somatic reversion of the BRCA2 frameshift deletion mutation occurred with PARP inhibitor treatment, accounting for the acquired resistance to PARP inhibition. Although this phenomenon has been described in case reports and small case series, the frequency of reversion mutations in BRCA1 and BRCA2 mutation carriers remains unknown; further studies are needed.

- Written by Elaine M Walsh and Cesar A Santa-Maria

\section{Soran A, Ozmen V, Ozbas S, et al. Randomized trial comparing resection of primary tumor with no surgery in Stage IV breast cancer at presentation: protocol MF07-01. Ann. Surg. Oncol. 25(11), 3141-3149 (2018).}

The prognostic effect of primary tumor resection for de novo Stage IV BC patients is controversial. This study is a multicenter, Phase III, randomized, controlled study comparing locoregional treatment (LRT) followed by systemic therapy (ST) with ST alone. A total of 274 Stage IV patients were randomized. Hazard of death was $34 \%$ lower in the LRT group than in the ST group (hazard ratio [HR]: 0.66; $p=0.005$ ). Estrogen receptor/progesterone receptor $(\mathrm{ER} / \mathrm{PR})(+)$, HER2/neu (-), patients younger than 55 years and patients with solitary bone-only metastases were significantly better factors for LRT. However, the 3-year survival rate that is the primary end point was similar for the LRT $(60 \%)$ and ST $(51 \%)$ groups $(\mathrm{p}=0.10)$. Moreover, the patients in the LRT group had higher rates of ER/PR positivity ( 85.5 vs $71.8 \%$; $\mathrm{p}<0.05$ ) and lower rates of triple-negative tumors ( 7.3 vs $17.4 \%$; $\mathrm{p}<0.05$ ) than the patients in the ST group.

The results suggested that the primary tumor resection have the possibility to prolong the survival for de novo stage IV BC. However, additional data is needed to confirm them.

- Written by Tadahiko Shien

\section{Moore HCF, Unger JM, Phillips KA, et al. Final analysis of the prevention of early menopause study (POEMS)/SWOG Intergroup S0230. J. Natl Cancer. Inst. ePub doi: 10.1093/jnci/djy185 (2018).}

This is the final report of POEMS, which is the international Phase III trial of goserelin administration during chemotherapy to reduce ovarian failure in premenopausal early ER and PgR-negative BC patients. A total of 218 patients (chemotherapy + goserelin [CG]: 105, chemotherapy [C]: 113) were randomized and evaluated. Five years cumulative incidence of pregnancy was $23.1 \%$ on the $C G$ arm and $12.2 \%$ on the $\mathrm{C}$ arm (adjusted odds ratio $=2.34 ; \mathrm{p}=0.03)$. There was no significant difference in overall survival $(\mathrm{HR}=0.45 ; \mathrm{p}=0.06)$ and disease-free survival $(\mathrm{HR}=0.55 ; \mathrm{p}=0.09)$ between two arms. Goserelin was effective to avoid premature menopause and to become pregnant without adverse effect on prognosis for premenopausal patients with ER and PgR-negative early breast cancer.

- Written by Tadahiko Shien

Balasubramanian I, Fleming CA, Corrigan MA, Redmond HP, Kerin MJ, Lowery AJ. Meta-analysis of the diagnostic accuracy of ultrasound-guided fine-needle aspiration \& core needle biopsy in diagnosing axillary lymph node metastasis. Br. J. Surg. 105(10), 1244-1253 (2018).

The results of meta-analysis of the diagnostic accuracy of ultrasound-guided fine-needle aspiration (US-FNA) and core needle biopsy (US-CNB) in diagnosing axillary lymph node metastasis of breast cancer were reported. A total of 1353 patients from six studies were included. The sensitivity in diagnosing axillary lymph node metastasis of US-FNA and US-CNB were 88 and 74\%, respectively. Both US-FNA and US-CNB had 100\% specificity. The complication (pain, hematoma and bruising) rates were significantly higher for US-CNB $(7.1 \%$; p < 0.001) and the requirement for repeat diagnostic procedure (insufficient sample, suspicious or indeterminate histology) was significantly greater for US-FNA $(4.0 \%$; $<<0.001)$. US-CNB is a superior diagnostic procedure for axillary staging in BC, especially to determine the indication of neoadjuvant chemotherapy.

- Written by Tadahiko Shien

Financial \& competing interests disclosure

The authors have no relevant affiliations or financial involvement with any organization or entity with a financial interest in or financial conflict with the subject matter or materials discussed in the manuscript. This includes employment, consultancies, honoraria, stock ownership or options, expert testimony, grants or patents received or pending, or royalties. 
No writing assistance was utilized in the production of this manuscript.

\section{Open access}

This work is licensed under the Attribution-Non Commercial-No Derivatives 4.0 Unported License. To view a copy of this license, visit http://creativecommons.org/licenses/by-nc-nd/4.0/ 
